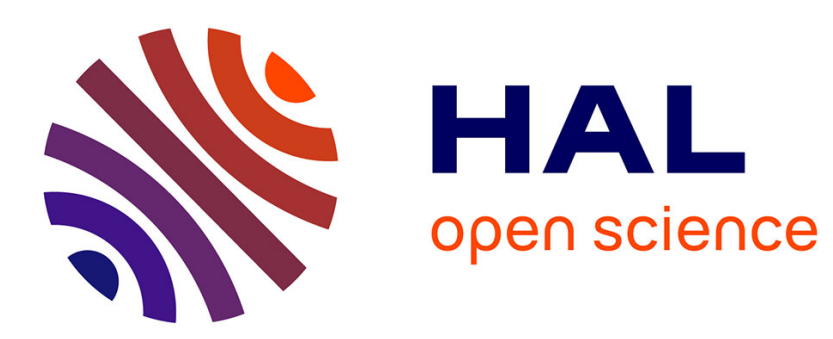

\title{
The Equilibrium Orientation of Scalar Gradient in a Lagrangian Model of Three-dimensional Turbulence
}

Michel Gonzalez

\section{To cite this version:}

Michel Gonzalez. The Equilibrium Orientation of Scalar Gradient in a Lagrangian Model of Three-dimensional Turbulence. Journal of the Physical Society of Japan, 2019, 88 (8), pp.085001. 10.7566/JPSJ.88.085001 . hal-02181450

\section{HAL Id: hal-02181450 \\ https://hal.science/hal-02181450}

Submitted on 12 Jul 2019

HAL is a multi-disciplinary open access archive for the deposit and dissemination of scientific research documents, whether they are published or not. The documents may come from teaching and research institutions in France or abroad, or from public or private research centers.
L'archive ouverte pluridisciplinaire HAL, est destinée au dépôt et à la diffusion de documents scientifiques de niveau recherche, publiés ou non, émanant des établissements d'enseignement et de recherche français ou étrangers, des laboratoires publics ou privés. 


\title{
The Equilibrium Orientation of Scalar Gradient in a Lagrangian Model of Three-dimensional Turbulence
}

\author{
Michel Gonzalez \\ Centre National de la Recherche Scientifique, Unité Mixte de Recherche 6614/CORIA \\ Site universitaire du Madrillet, 76801, Saint-Etienne du Rouvray, France \\ The kinematics of scalar gradient in three-dimensional turbulence is revisited using \\ stochastic Lagrangian modelling. The equilibrium direction is computed numerically. \\ It noticeably departs from the compressional direction, and is the attracting - and \\ preferential - alignment for the scalar gradient.
}

The kinematics of the gradients of scalars - such as temperature or concentration is relevant to the dynamics of vectors. ${ }^{1)}$ More specifically, it is connected to the basic mechanisms of small-scale mixing, and is thereby relevant to many fields - reacting flows, geophysical flows, etc. Scalar gradient orientation, in particular, plays a determinig role in stretching and thus in the mixing process. In this study, it is analysed by accounting for the existence of an equilibrium direction.

The stable fixed point of the orientation equation of a scalar gradient - referred to as the equilibrium direction - is in general different from the compressional direction and is thus a special direction to consider in addition to the strain principal axes. ${ }^{2-4)}$ At infinite Péclet number, this direction - provided it exists - results from the opposed effects of strain and rotation. It was derived analytically in the two-dimensional case, for a nondiffusive scalar gradient. ${ }^{2)}$ The three-dimensional case is analytically tractable assuming vorticity aligned with a strain eigenvector. ${ }^{3,4)}$ In the present work, this assumption is relaxed and the equilibrium direction is determined numerically in the general threedimensional case.

A stochastic Lagrangian model is used to simulate three-dimensional isotropic turbulence, and to derive the alignment statistics of the scalar gradient. The modelled equation for the velocity gradient tensor, $\mathbf{A}$, is: ${ }^{5,6)}$

$$
d \mathbf{A}=\left(-\mathbf{A}^{2}+\frac{\operatorname{Tr}\left(\mathbf{A}^{2}\right)}{\operatorname{Tr}\left(\mathbf{C}_{\tau_{\eta}}^{-1}\right)} \mathbf{C}_{\tau_{\eta}}^{-1}-\frac{\operatorname{Tr}\left(\mathbf{C}_{\tau_{\eta}}^{-1}\right)}{3 T} \mathbf{A}\right) d t+\sqrt{\frac{2}{T}} d \mathbf{W}
$$

with $T$, the integral time scale, and $\mathbf{C}_{\tau_{\eta}}$ a model for the Cauchy-Green tensor, $\mathbf{C}_{\tau_{\eta}}=$ 
$\exp \left(\tau_{\eta} \mathbf{A}\right) \exp \left(\tau_{\eta} \mathbf{A}^{T}\right)$, where $\tau_{\eta}$ is the Kolmogorov time scale; $d \mathbf{W}$ is the increment of a tensorial Wiener process. The equation for the scalar gradient, $\mathbf{G}$, is: ${ }^{4)}$

$$
d \mathbf{G}=-\left(\mathbf{A}^{T} \mathbf{G}+\frac{\operatorname{Tr}\left(\mathbf{C}_{\tau_{\eta}}^{-1}\right)}{3 T_{\theta}} \mathbf{G}\right) d t+\sqrt{\frac{2}{T_{\theta}}} d \mathbf{W}_{\mathbf{G}}
$$

where $T_{\theta}$ is assumed to coincide with the transfer time scale of scalar variance; $d \mathbf{W}_{\mathbf{G}}$ is the increment of a Wiener process. In Eqs. (1) and (2), stretching is exactly accounted for. Second term of Eq. (1) is a model for the pressure Hessian. Viscous effects - third term of Eq. (1) - and molecular diffusion - second term of Eq. (2) - are modelled as proposed by Jeong and Girimaji. ${ }^{7)}$

Time scales are normalised by the integral time scale $(T=1)$. The Kolmogorov time scale is prescribed as $\tau_{\eta}=0.1$ - which corresponds to a Taylor microscale Reynolds number, $\mathrm{Re}_{\lambda}$, close to $150 .{ }^{6)}$ Linear modelling of molecular diffusion makes scalar gradient orientation independent of diffusive effects; the predicted alignment properties are therefore those of high-Péclet-number scalar turbulence. Consistently, the scalar integral time scale is taken such that $T_{\theta} / T=1$, a value derived for Schmidt number greater than unity. ${ }^{8)}$ The orientation of $\mathbf{G}$ may be sensitive to $T_{\theta}$ because of randomization caused by the forcing term in $T_{\theta}^{-1 / 2}$, but this relatively large value of $T_{\theta} / T$ - as compared to $T_{\theta} / T \sim 0.3$ found for small Schmidt number ${ }^{8)}$ - curbs this effect. Equations (1) and (2) are solved using a second-order predictor-corrector scheme. The calculation is run with time step $10^{-2}$ for a total time ranging from $2 \times 10^{5} \mathrm{~T}$ to $2 \times 10^{7} \mathrm{~T}$, depending on the sample length needed for statistics.

Neglecting molecular diffusion, the equation for the director cosines, $n_{i}=\mathbf{G} \cdot \mathbf{e}^{(i)} /|\mathbf{G}|$, of the scalar gradient in the strain basis is:

$$
\frac{D \mathbf{n}}{D t}=\boldsymbol{\sigma} \cdot \mathbf{n}+\mathbf{R} \times \mathbf{n},
$$

where $\boldsymbol{\sigma}=(\mathbf{n} \cdot \boldsymbol{\Lambda} \cdot \mathbf{n}) \mathbf{I}-\boldsymbol{\Lambda}$ includes the effect of stretching, and $\mathbf{R}=\boldsymbol{\omega} / 2-\boldsymbol{\Omega}$ is the effective rotation rate. Vectors $\mathbf{e}^{(i)}$ are the eigenvectors of the strain tensor, with $i=1$ for expansion, $i=2$ for 'intermediate' direction, and $i=3$ for compression; $\Lambda$ is defined by $\Lambda_{i j}=\lambda_{i}$ for $i=j$, and 0 otherwise; the $\lambda_{i}$ 's are the strain eigenvalues - with $\lambda_{1}>0$, $\lambda_{3}<0$, and $\lambda_{1}+\lambda_{2}+\lambda_{3}=0$; vector $\boldsymbol{\omega}$ is vorticity, and $\boldsymbol{\Omega}$ is the rotation rate of the strain basis. ${ }^{9)}$

The instantaneous equilibrium direction - in the Lagrangian view : the local equilibrium direction - is defined by vector $\mathbf{n}^{(e q)}$. The latter determines the orientation the scalar gradient would take if its response to the variations of the velocity gradient tensor 
were infinitely fast. This reference direction is computed at each time step by solving the non-linear algebraic system given by:

$$
\boldsymbol{\sigma} \cdot \mathbf{n}+\mathbf{R} \times \mathbf{n}=0
$$

which expresses that the equilibrium direction results from the balance between strain, which tends to bring the scalar gradient on the compressional strain principal axis, and effective rotation which, in contrast, constantly moves it away. The numerical data for which a stable equilibrium direction exists define a subset that is referred to as the equilibrium sample. If $\mathbf{A}$ is restricted to a Gaussian process, the equilibrium sample amounts to $50 \%$ of the total flow; in the turbulent case, for which $\mathbf{A}$ is derived from Eq. (1), this percentage is $63 \%$. In the special cases where vorticity aligns with a strain eigenvector, the average error between the numerical solution and the analytic solution ${ }^{3,4)}$ is $0.27^{\circ}$ at most.

The equilibrium direction is essentially normal to $\mathbf{e}^{(2)}$ (Fig. 1) and thus lies on the plane $\left(\mathbf{e}^{(1)}, \mathbf{e}^{(3)}\right)$. It does not coincide with the compressional strain principal axis; this would occur for pure strain $(\mathbf{R}=0)$ only. The probability density function (p.d.f) of angular gap between $\mathbf{e}^{(3)}$ and $\mathbf{n}^{(e q)}$ peaks at $23^{\circ}$ - which is also roughly the average angular gap.

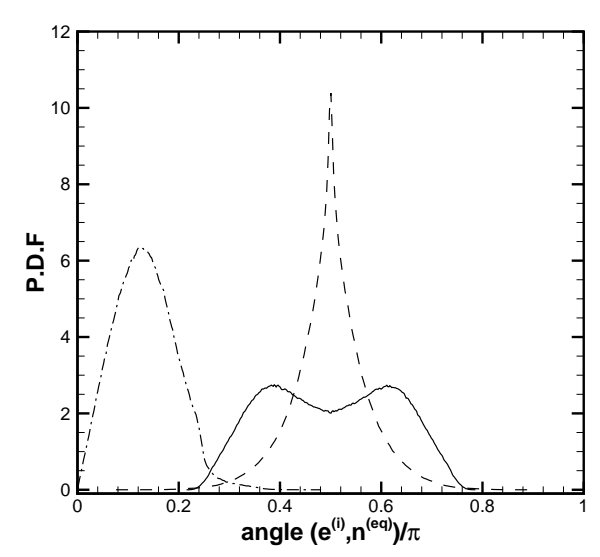

Fig. 1. P.d.f's of angular gap between equilibrium direction and strain eigenvectors, $\mathbf{e}^{(i)}$; solid line: $i=1$; dashed line: $i=2$; dash dot line: $i=3$.

Scalar gradient alignments corresponding to cosine greater than 0.95 occur closer to the equilibrium direction than to the compressional one (Fig. 2). By accounting for rotation in addition to strain, the equilibrium direction captures a more realistic 
kinematics of the scalar gradient. This finding backs up the results derived in the special case where vorticity is aligned with a strain axis. ${ }^{4)}$ Better still, the preferential alignment of scalar gradient predicted in two-dimensional turbulence ${ }^{2)}$ is thereby confirmed in the three-dimensional case.

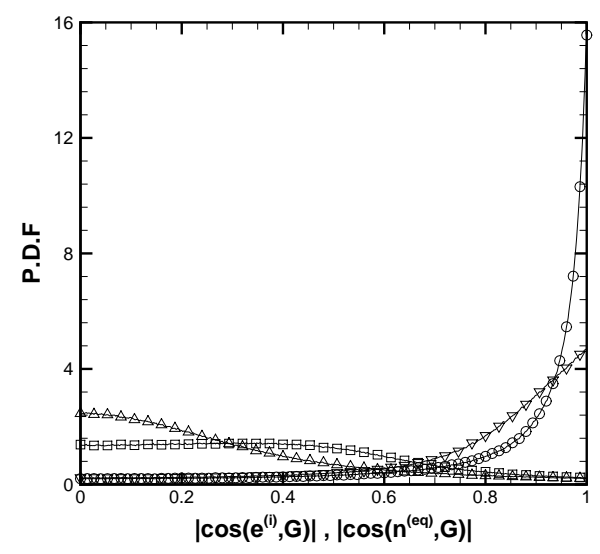

Fig. 2. P.d.f's of $\left|\cos \left(\widehat{\mathbf{e}^{(i)}, \mathbf{G}}\right)\right|$ (squares: $i=1$; deltas: $i=2$; gradients: $\left.i=3\right)$ and of $\mid \cos \left(\widehat{\left.\mathbf{n}^{(e q)}, \mathbf{G}\right)} \mid\right.$ (circles) in the equilibrium sample.

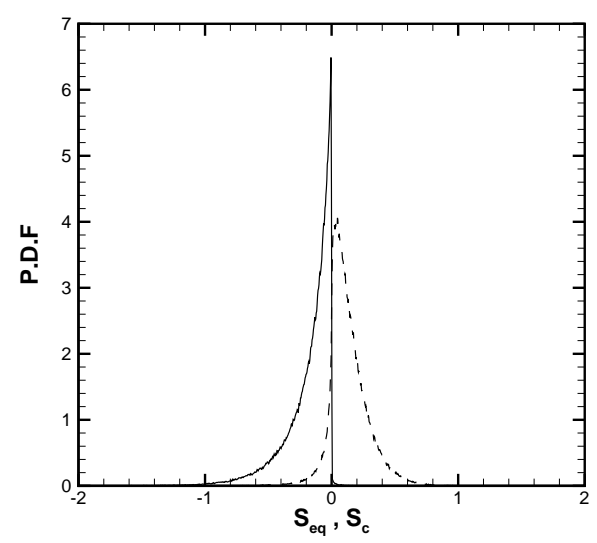

Fig. 3. P.d.f's of $S_{e q}$ (solid line) and $S_{c}$ (dashed line).

As shown in a two-dimensional flow, ${ }^{10}$ the equilibrium direction is the attracting alignment for the scalar gradient. This feature is checked in the equilibrium sample by comparing $S_{e q}=-\operatorname{sgn}\left\{\cos \left(\widehat{\mathbf{n}^{(e q)}, \mathbf{G}}\right)\right\} \cdot F_{e q}$ and $S_{c}=-\operatorname{sgn}\left\{\cos \left(\widehat{\mathbf{e}^{(3)}, \mathbf{G}}\right)\right\} \cdot F_{c}$, where $F_{e q}=(\boldsymbol{\sigma} \cdot \mathbf{n}+\mathbf{R} \times \mathbf{n}) \cdot \mathbf{n}^{(e q)}$ is the rate of change of $\cos \left(\widehat{\mathbf{n}^{(e q)}, \mathbf{G}}\right)$, and $F_{c}=(\boldsymbol{\sigma} \cdot \mathbf{n}+\mathbf{R} \times$ 
$\mathbf{n}) \cdot \mathbf{e}^{(3)}$ is the rate of change of $\cos \left(\widehat{\mathbf{e}^{(3)}, \mathbf{G}}\right)$; negativity of $S_{e q}\left(\right.$ resp. $\left.S_{c}\right)$ means attraction towards equilibrium (resp. compressional) direction. Figure 3 shows the p.d.f's of $S_{e q}$ and $S_{c}$ - respectively, $P_{e q}\left(S_{e q}\right)$ and $P_{c}\left(S_{c}\right)$ - conditioned on the direction of $\mathbf{G}$ being in the right circular cone the aperture of which is $\left(\mathbf{e}^{(3), \mathbf{n}^{(e q)}}\right)$. Negative values of $S_{e q}$ are obviously much more frequent than those of $S_{c}: A_{e q}^{-}=\int_{-\infty}^{0} P_{e q}\left(S_{e q}\right) d S_{e q}=0.98$, while $A_{c}^{-}=\int_{-\infty}^{0} P_{c}\left(S_{c}\right) d S_{c}=0.12$; clearly, rather than the compressional direction, it is the equilibrium direction that draws the scalar gradient.

Those findings show the existence of an equilibrium direction of the scalar gradient that is definitely distinct from strain eigenvectors. Strongest alignments occur rather with the equilibrium direction than with the compressional one, as already shown in two-dimensional turbulence. The attracting direction for the scalar gradient orientation is the equilibrium direction, and not the compressional direction as is usually stated. As the model was previously shown to retrieve essential features of both velocity gradient and scalar gradient tensors, ${ }^{4,6)}$ the present results are likely to reasonably simulate realistic kinematics of scalar gradient. They would still deserve to be compared, for instance, to DNS data. 


\section{References}

1) P. G. Saffman, J. Fluid Mech. 16, 545 (1963).

2) G. Lapeyre, P. Klein, and B. L. Hua, Phys. Fluids 11, 3729 (1999).

3) A. Garcia and M. Gonzalez, Phys. Fluids 18, 058101 (2006).

4) M. Gonzalez, Phys. Fluids 21, 055104 (2009).

5) L. Chevillard and C. Meneveau, Phys. Rev. Lett. 97, 174501 (2006).

6) L. Chevillard, C. Meneveau, L. Biferale, and F. Toschi, Phys. Fluids 20, 101504 (2008).

7) E. Jeong and S. S. Girimaji, Theor. Comput. Fluid Dyn. 16, 421 (2003).

8) P. K. Yeung, Shuyi Xu, and K. R. Sreenivasan, Phys. Fluids 14, 4178 (2002).

9) E. Dresselhaus and M. Tabor, J. Fluid Mech. 236, 415 (1992).

10) M. Gonzalez, Theor. Comput. Fluid Dyn. 32, 521 (2018). 